\title{
ISOMETRIES OF THE TRACE CLASS
}

\author{
BERNARD RUSSO ${ }^{1}$
}

Let $J$ denote the Banach space of trace class operators on a complex Hilbert space $H$, in the norm $\|T\|_{1}=\operatorname{Tr}(|T|)$. The space $J$ is a two-sided ideal in the algebra $\mathscr{L}$ of all bounded operators on $H$. See [4].

THEOREM. If $\Phi$ is a linear isometry of the Banach space J onto itself, then there exists $a^{*}$-automorphism or $a^{*}$-antiautomorphism $\alpha$ of $\mathfrak{L}$ and a unitary operator $U$ in $\mathfrak{L}$ such that $\Phi(T)=\alpha(T U),(T$ in $J)$.

REMARK 1. The theorem provides a partial answer to [3, Remark 1, p. 231].

Proof. The adjoint $\Phi^{\prime}$ is a linear isometry of $\mathscr{L}$ onto $\mathscr{L}$ so by results of Kadison [2, Theorem 7, Corollary 11] has the form $\Phi^{\prime}(A)$ $=U \alpha(A)$ where $\alpha$ and $U$ are as described in the statement of the theorem. It is elementary that $\Phi(T)=\Psi(T U)$ where $\Psi^{\prime}=\alpha$. The proof will be complete if it is shown that $\alpha$ is the adjoint of $\alpha^{-1}$ (restricted to $\Im$ ). By the folk result $[1$, pp. 256, 9] it is sufficient to check this in the following two cases:

(i) $\alpha(A)=V A V^{-1}$ with $V$ a fixed unitary operator; then $\langle T, \alpha(A)\rangle$ $=\left\langle T, V A V^{-1}\right\rangle=\left\langle V^{-1} T V, A\right\rangle=\left\langle\alpha^{-1}(T), A\right\rangle$,

(ii) after the choice of an orthonormal basis, $\alpha(A)$ is the transposed matrix of $A$; then $\langle T, \alpha(A)\rangle=\operatorname{Tr}(T \alpha(A))=\operatorname{Tr}(\alpha(T) A)=\left\langle\alpha^{-1}(T), A\right\rangle$.

REMARK 2. A previous version of the above proof exploited a knowledge of the extreme points of the unit sphere of $J$. These were determined to be the partial isometries with initial (hence final) domain one-dimensional.

\section{REFERENCES}

1. J. Dixmier, Les algèbres d'opérateurs dans l'espace hilbertien, Gauthier-Villars, Paris, 1957.

2. R. V. Kadison, Isometries of operator algebras, Ann. of Math. (2) 54 (1951), $325-338$.

3. B. Russo, Isometries of $L^{p}$ spaces associated with finite von Neumann algebras, Bull. Amer. Math. Soc. 74 (1968), 228-232.

4. R. Schatten, Norm ideals of completely continuous operators, Springer-Verlag, Berlin, 1960.

University of CALIFornia, Irvine

Received by the editors March 7, 1969.

1 This research was supported by the National Science Foundation Grant GP. 8291. 